\title{
nature
}

cell biology

\section{Thank you !}

'We would like to take

this opportunity to

thank everyone who

has helped Nature Cell

Biology.'
M

uch has been written about the benefits and pitfalls of the peer review process, and despite its shortcomings, no superior alternative has been found. No one would deny that the review process is crucial in improving papers, by providing different viewpoints and interpretations to ensure that published papers meet the required scientific standards. Peer review is therefore an integral part of scientific progress.

Above all, peer review relies on the willingness of scientists to participate in the process, and to provide fair and constructive comments that improve papers. For this reason, we would like to take this opportunity to thank everyone who has helped Nature Cell Biology, for their time and effort over the past three years. Like all Nature titles, Nature Cell Biology does not have an editorial board, and decisions on which papers are sent out for review are made by the editorial team of the journal. However, we frequently consult with experts in the field to reach these decisions, and we would also like to extend our thanks to those who have provided us with such helpful and informed advice.

\section{AN IMPORTANT CHOICE}

Every editor of a scientific journal will agree that the outcome of the review process depends, to a considerable extent, on the choice of reviewers. The editors of Nature Cell Biology do not take this responsibility lightly. We strive to consult those researchers who in the past have provided critical, yet fair, well-informed and constructive reports. In addition, we continuously add scientists to our pool of referees, including both junior, upcoming scientists, as well as more established figures. An important part in developing such a wide-ranging panel comes from valuable suggestions from the scientific community, but also through meeting new potential reviewers at conferences, or during lab visits. By spreading the review process out in this way, and not limiting the scope of individuals whose advice we seek to a set editorial board, we hope to avoid undue control over a subject area by a selected few. This should ensure the representation of a diversity of viewpoints and expertise. In return, we hope that this allows the cell biology community to participate in shaping the representation of their field in the journal.

As we appreciate the time and effort our reviewers put in, we will continue to provide referees with feedback, informing them about the comments of other reviewers and the decision taken on a paper. The feedback we have received for this service (such as "I find it really helpful - it allows me to more effectively calibrate my comments") suggest that this information is much appreciated. We look forward to a productive collaboration with both our 'old' and 'new' reviewers in 2002. 